\title{
Persuasive and Culture-aware Feedback Acquisition
}

\author{
Malik Almaliki ${ }^{1}$ and Raian $\mathrm{Ali}^{2}$ \\ ${ }^{1}$ Taibah University, Medina, KSA \\ mrmalki@taibahu.edu.sa \\ ${ }^{2}$ Bournemouth University, Bournemouth, UK \\ rali@bournemouth.ac.uk
}

\begin{abstract}
User feedback is an important factor to improve software quality. For example, it can provide information on missing features and clarify user trends and preferences for future improvement. However, gathering user's feedback is not an easy process since the majority of users lack motivation and interest in providing feedback, especially in a constant and frequent style. In addition, studies have noted that the cultural differences among users also play a role in affecting their motivations to feedback acquisition. In this paper, we empirically investigate the role of culture in affecting users' perception and motivations to give feedback. Our study identifies some key differences between Western and Middle Eastern users on what motivate them to provide feedback and what could have an influence on the quality of the feedback they give. This also makes the case for the need to design a persuasive and culture-aware feedback acquisition.
\end{abstract}

Keywords: User's Feedback, Persuasive Technology, Software Engineering

\section{Introduction}

In the context of software system, the software's ability to adapt to different cultures in users' space is important for improving its success in wider contexts and constructs a key requirement for professional and ethical reasons [1]. Generally speaking, most software designs follow a western cultural cues. This has caused a design gap when users coming from different cultures (i.e. eastern cultures) use the software within their cultural context. An example is clearly seen in the different ways people from all over the world use social networks. This is perhaps due to the fact that software industry is largely led by western management and developers [2]. Therefore, software systems that are marketed worldwide need to be tailored to fit the different cultures [3] as designs that are successful in one culture may fail dramatically in others $[4,5]$.

User's feedback constructs an important source for information needed for planning software evolution and adaptation [6]. This means that users need to be persuaded to provide feedback frequently. However, persuading users is not an easy task as the majority of users have little motivation and interests in providing such feedback in an ongoing style [7]. Moreover, motivating users would highly depend on their culture and values. This sheds the lights on the potential use of persuasive technology in order to empower users' willingness to give feedback thus their experience and software's success. Persuasive technology refers to the technology that is designed to change the atti- 
tudes or behaviors of users through persuasion and social influence, but not through coercion [8]. It has been used as an effective approach to increase users' engagement in many areas including the area of human-computer interaction. Recent research indicates that persuasive technology is more effective when it is tailored to the culture of its intended target audience [9]. However, little research has explicitly investigated the relationship between persuasive technology and culture [10].

In this paper, the authors qualitatively (using a focus group approach) study the effect and impact of cultural backgrounds on users' social motives to give feedback. The study's focus is on two different cultural backgrounds: Middle Eastern users and European users. The paper also advocates the need to design the acquisition process taking into account the persuasion goal of feedback acquisition in different cultures. The results of this study are meant to help devising a persuasive and systematic method for conducting a socially aware feedback acquisition that can adapt to different types of users in terms of their cultural backgrounds (Middle Eastern and European users). This will ultimately maximize feedback quality, users' satisfactions and motivations to give feedback.

The paper is structured as follows. In Section 2 we briefly discuss feedback acquisition and its relation to cultural backgrounds. In Section 3 we describe the research method adopted. In Section 4 we present and discuss the results of our study and in Ssection 5 we present our conclusion.

\section{Feedback Acquisition and Cultural differences}

In [7] the authors conducted an empirical study to investigate users' behaviour to feedback requests and discover what motivates them to provide feedback. The study consisted of two phases: a qualitative and a quantitative phase. In the qualitative phase 7 interviews were conducted whereas in the quantitative phase, a survey was used and 100 responses were collected. The results of their study showed a preliminary indication that the variant cultural backgrounds of users has a noticeable impact on their behaviour and how they are socially motivated to give feedback. The preliminary results and survey questions related this aspect are provided in [22].

While a number of researchers have already investigated cultural differences in relation to software such as how graphics, language, object formatting, colours, and layout of web sites and other user preferences is perceived in different cultures [11, 12, $13,14]$, to the our best knowledge no studies have yet investigated how users with different backgrounds behave in response to feedback acquisition and how their culture frame affect their motivations to feedback requests. This paper aims at qualitatively investigating this aspect to help improving the design of a persuasive and culture-aware feedback acquisition.

\section{Research Method}

In empirical research, researchers might need to qualitatively follow up or build upon quantitative results for the purpose of explaining or further investigating the quantitative results [15]. This design is suitable for researchers who need qualitative data to explain significant, non-significant or surprising quantitative results [16, 17]. The 
results discussed in [7] indicated that the variant cultural backgrounds of users have a noticeable impact on users' behaviour and how they are socially motivated to give feedback. This was only an indicator which would need confirmation and clarification. To achieve that, this study follows up the quantitative results in [7] with a qualitative phase through focus groups which is a powerful tool to get insights and stimulate discussions in a small group of participants.

A focus group is a qualitative research method in which a group of people are gathered to be asked about their opinions, beliefs, or attitudes regarding an issue, phenomena, service, etc. The questions are asked in an interactive setting which allows participants to talk freely about their thoughts to other group members. Due to the nature of the study where social/group interaction is needed and the type of the asked questions which mainly relate to the culture impact (Middle Eastern and European cultures) on social perception of feedback acquisition of both the feedback provider and those who watch it, this paper adopted Focus Group as a data collection method in this investigatory study.

\subsection{Focus Group Design}

Four Semi-structured focus groups were conducted in two countries (Saudi Arabia and the UK) with 27 participants to further explore how Middle Eastern and European users behave in response to feedback acquisition and how their culture frame affect their motivations to feedback requests. Participants were carefully selected in order to guarantee a high level of diversity and to avoid bias (e.g. various age groups, backgrounds and gender). The first two focus groups took place on June-2014 in the UK and were conducted with European participants to investigate how their culture impacts their perception to feedback acquisition. Whereas the other two focus groups were held on July-2014 and conducted with Saudi participants to investigate the Middle Eastern culture's impact to feedback acquisition. Each focus group session lasted for about an hour which makes an amount of four hours in total.

The focus groups protocol was developed in the light of the results discussed in [7] in which an indication to cultural differences between European and Middle Eastern users on what motivate them to provide feedback was discovered. In particular, the four social factors (Feedback acquisition as a social activity, Social recognition, Volume of already given feedback and Visibility and similarity of others feedback) that influence how Middle Eastern and European users are socially motivated to give feedback served as a foundation to develop the protocol of the focus group. The focus group protocol is available in [22].

The protocol was iteratively reviewed and revised by 3 researchers to ensure clarity and understandability. Participants were briefed to the session and the discussed topic through a 10 minutes presentation in which some example of feedback acquisition in software application were also given to more familiarise the participants with the discussed topic. Each participant received $£ 15$ amazon vouchers as an appreciation for taking part in the study. 


\subsection{Sampling}

Purposeful sampling is a common technique in qualitative research [18]. In this study, purposeful sampling was used to recruit the participants. The inclusion criteria of this study allowed for participants who are European or were born and raised in Europe to take part in the first two focus groups dedicated to study European people (7 participants for the first focus group and 6 for the second). On the other hand, participants who are Saudis or were born and raised in Saudi Arabia were recruited to take part in the other two focus groups dedicated to study Middle Eastern people in which 7 participants took part in each focus group.

In addition, the inclusion criteria allowed for participants within an age range of 18 to 71 and average computer users who use typical and diverse set of popular software applications rather than domain specific software for everyday life activities. This sampling criterion were developed to allow for more variety in selecting participants and reflecting users' experience with popularly used software applications. This can maximize the generalizability of the results. For more details about the participants' characteristics (e.g. age, gender, home country, etc) please refer to [22].

The authors assumed that Saudi users could be a good fit to represent the Middle Eastern culture. It is due to the fact that Saudi Arabia is one of the largest countries in the Middle East and could fairly represent users' cultures in the region when it comes to the use of software. Statistics indicate that Saudi Arabia has over 6 million active Facebook users which is the highest Facebook user rate in the region. With more than 3 million active Twitter users, Saudi Arabia takes the lead not only in the Middle East, but in the world in its Twitter users' growth rate. In addition, More than 90,000,000 videos are watched daily on YouTube in Saudi Arabian which is more than any daily YouTube video views number in the world [19]. Based on this statistics, the available resources to the authors and time, the authors assumes that Saudi Arabia is a reasonable fit to be adopted in this study to represent Middle Eastern culture to feedback acquisition.

\subsection{Analysis}

Focus groups were audio taped and transcribed verbatim analysis was performed in several steps which included: (1) initial exploration of the gathered data by reading the transcripts; (2) coding data by labelling and segmenting the text; (3) using codes to generate themes by gathering similar codes together; (4) connecting, comparing and interrelating themes. Credibility of the findings was maximized by using an inter-coder agreement check and academic advisor's auditing [18, 20].

\section{$4 \quad$ Findings}

As previously stated, the focus group design covered how a number of social factors influence Middle Eastern and European users with regard to feedback acquisition. In this section, these social factors are used to structure, represent and discuss the main themes of the findings. The themes and codes that highlight the encountered 
behavioural differences between the two studied groups (Middle Eastern and European users) to feedback acquisition are shown in Table 1 .

Table 1. A breakdown of the themes and codes of the analysis.

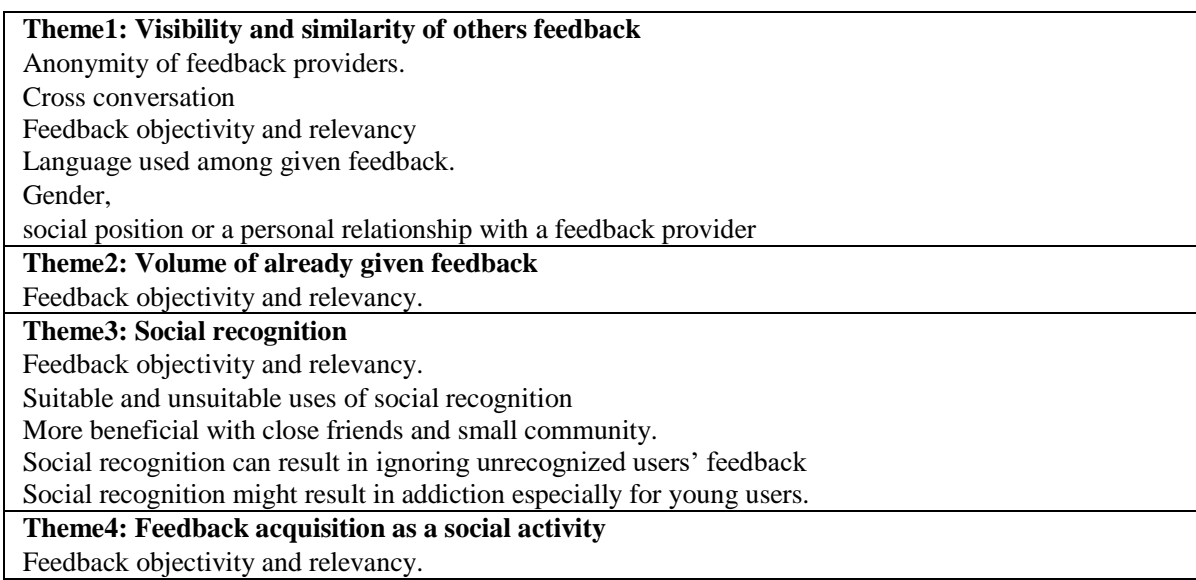

\subsection{Visibility and Similarity of Others Feedback}

Generally speaking, the responses from Middle Eastern participants and European participants are noticeably different when they were asked whether the visibility of others feedback (the ability to others feedback before giving feedback) and the similarity of their feedback to others feedback would have effect on their willingness to give feedback. Although feedback visibility plays a role in motivating both Middle Eastern and European participants to give feedback, Middle Eastern participants seemed to be more concerned and socially motivated by this factor than European participants. This trend became more obvious when they were asked whether knowing the similarity of their feedback to others would affect their willingness to give feedback. One the participants mentioned in this regard "I'm normally interested in replying to reviews that I do not agree with". Figure 1 gives a general view of how the different cultures' impact on this social factor could affect the quality of given feedback. In addition, the following dimensions of this theme were extracted from the participants' responses:

Anonymity of feedback providers. Users ability to give feedback anonymously (i.e. using nicknames instead of the real names) is shown to play a role in motivating both parties (Middle Eastern and European users) to give feedback especially when the given feedback is publicly open and seen by other users. This is perhaps due to several factors (i.e. dissociative anonymity, invisibility, solipsistic introjection, dissociative imagination, and minimization of authority) as discussed in [7] which allow users to enjoy more freedom in expressing their opinion about a product or a software service. One of the participants said "I would be more interested in truly engaging when no one knows me. It feels like you are free to say whatever you like and you will still be unknown". However, anonymity does not seem to be a motivating factor when a feedback provider is part of an online closed community such as a Facebook group. In general, users prefer to know the identity of the feedback provider in their closed social 
network/community since it makes them more comfortable participating and discussing a software service or a product.

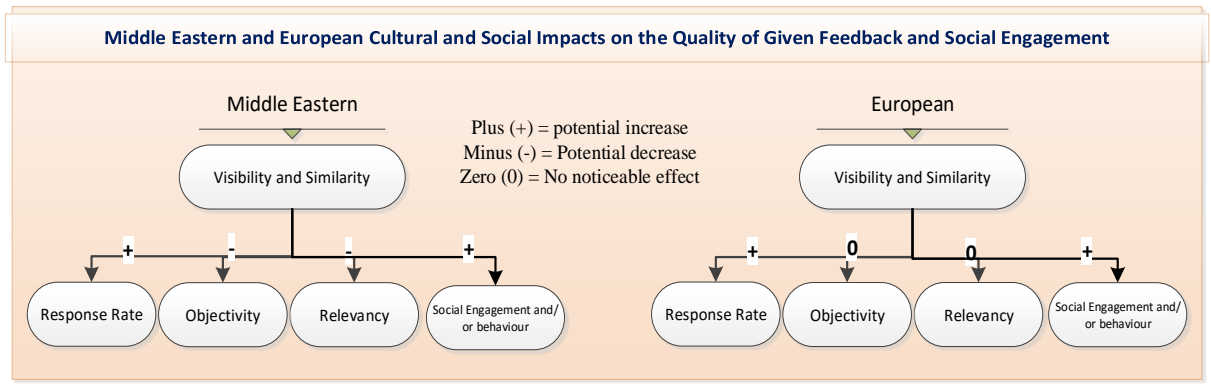

Fig. 1. The effect of feedback visibility and similarity in Middle Eastern and European culture.

On the downside, anonymity seems to affect the objectivity and relevancy of provided feedbacks by both parties but it is more prominent with the Middle Eastern than European users. Their overall responses indicated that anonymity could results in the so-called online disinhibition effect [21] which gives users space to escape from their social constrains. However, this feeling of freedom can result in an overexpression and less objectivity and relevancy of their opinion about a service or a product. One of the participants commented "The problem is that some people think they can say anything or be unfair or even harm others because nobody knows them. That's not the point of being anonymous". Figure 2 gives a clearer view of how the different cultures' impact on this social factor could affect the quality of given feedback.

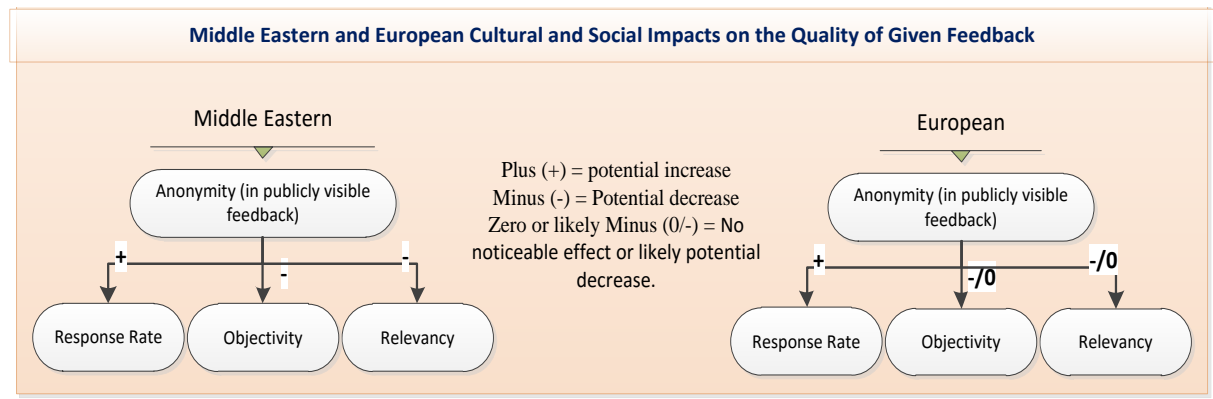

Fig. 2. The effect of anonymity on feedback in Middle Eastern and European culture.

Cross conversation, feedback objictivety and relevancy and language used. In the context of cross conversation (irrelevant feedback) and how it affects the provided feedback, users' responses indicated that feedback given by European users might enjoy a slightly higher degree of relevancy and objectivity than the feedback given by Middle Eastern users. This is perhaps due to the fact that Middle Eastern users are more socially engaged which could put some constrains on the relevancy and objectivity of their given feedback However, in all cases cross conversation (irrelevant feedback), subjectivity and the harshness degree in the language used among already given feedback can results in a low response rate by both parties (Middle Eastern and 
European users). It can also result in a harm to the software product or the provided service.

An example of this was given by one of the participants "if the cross conversations or the harshness of the used language leverage among users feedback such as two large groups of users fighting around irrelevant specific religious or political party, this can highly result in users from either group degrading and disliking the service or the product just to cause a harm to the service provider who they think he might be from the opposite party". Figure 3 gives a clearer view of how the different cultures' impact on this social factor could affect the quality of given feedback.

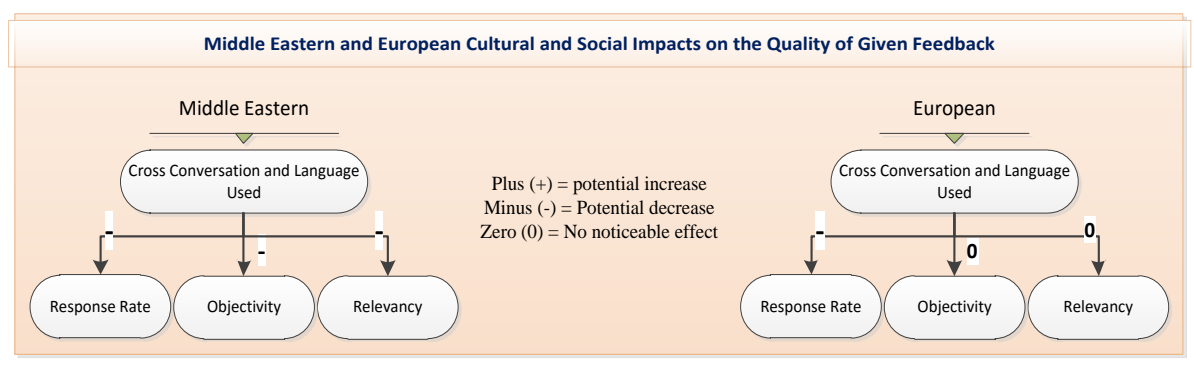

Fig. 3. The effect of cross conversation and language used on feedback in Middle Eastern and European culture.

Gender, social position or a personal relationship with a feedback provider. Being able to know/see the gender, social position or your personal relationship with a feedback provider gives a clear view about how users from the different two cultures (Middle Eastern and European cultures) are socially motivated to give feedback. In the European culture, users do not seem to be influenced by these factors to give feedback at all. This is perhaps due to the fact that they feel more socially- freewheeling than Middle Eastern users as described by some of the participants. A participant commented "I believe living in this community (European community) makes you less socially-dependent and do not easily accept to accomplish things with the help of your parents or friends for example. You always want to do things by yourself. This is how we grew up ".

On the other hand, users coming from Middle Eastern culture are significantly different to the European users in their perception of these factors. They feel highly motivated by these factors to give feedback especially with people/users they know (i.e. having a personal relationship with the person asking for feedback or the users giving feedback). Interestingly, the gender of a feedback provider is considered to be source of curiosity that motivates Middle Eastern users to give feedback which is not the case with the European users. Males/females would find it interesting to see how females/males think of a particular software service or product. One of the participants said "I would really love to see how males' feedback would be on a certain aspect of a software such as the interface colours. You know we love girly colours and this will always make my feedback clashes with males which is fun".

On the down side, motivating Middle Eastern users by these factors can result in a questionable quality of their given feedback in terms of its objectivity and relevancy to the discussed software service or product. Some of the Middle Eastern participants 
mentioned that they always tend to be supportive and on the side of their friends (i.e. software service provider who is asking for their feedback) regardless of the discussed product and their real opinion about it. Although this can be harmful to the quality of the feedback, it gives a nice example of a caring relationship among users. Figure 4 gives a clearer view of how the different cultures' impact on this social factor could affect the quality of given feedback.

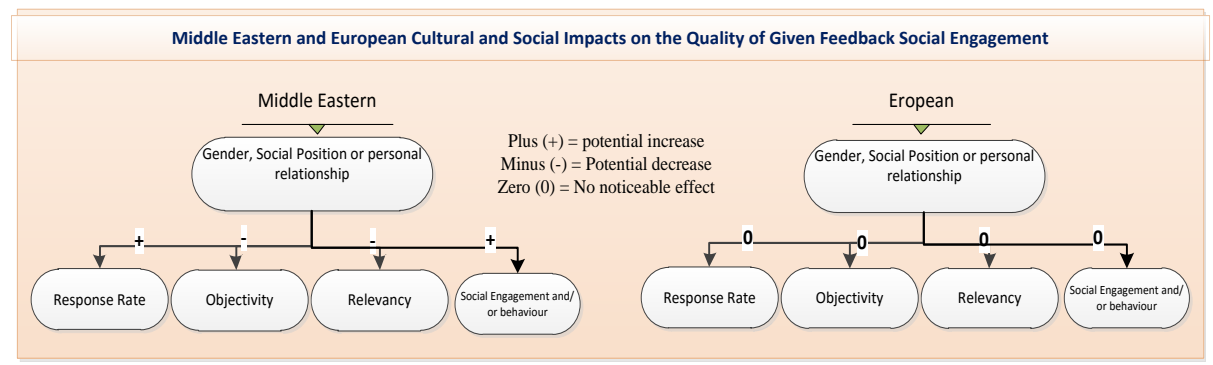

Fig. 4. The effect of gender, position or relationship on feedback in Middle Eastern and European culture.

\subsection{Volume of Already Given Feedback}

When participants were asked whether the number of feedback already provided on a software service or a product would affect their willingness to give feedback, Middle Eastern users showed a consensus on that they would like to provide feedback if there were only few feedback given rather than a large number of already given feedback. One of the participants commented "it makes me feel sorry when only few reviews are given especially when the app or service is good. I would certainly find a time to write my own review and help". In comparison, European users had a similar attitude but they perceive this as a less important factor when compared to Middle Eastern users.

Feedback objectivity and relevancy. The degree of feedback relevancy and objectivity given by both parties (Middle Eastern and European users) does not seem to be influenced by the volume of already given feedback. However, the low number of already given feedback can sometimes impose potential risk to the software service or product. Several users from both parties indicated that when a low number of feedback/reviews is already given it makes them lose interest in the provided service since it does not seem to be popular among users otherwise it would be highly reviewed by a large number of users. One of the participants commented "The first thing I do before downloading an app is to look at the number of reviews. A low number means to me less popular and useful". Figure 5 gives a clearer view of how the different cultures' impact on this social factor could affect the quality of given feedback.

\subsection{Social recognition}

Participants were asked whether being recognized by the community as feedback providers would affect their willingness to give feedback. The responses from the two groups were noticeably different. Users from Middle Eastern backgrounds indicated 
that being socially recognized as a feedback provider is an influential factor that could positively maximize their willingness to give feedback. There could be still some constraints on this, e.g., some participants commented that "it is nice to be visible only when others can see their feedback which led to some changes on the system".

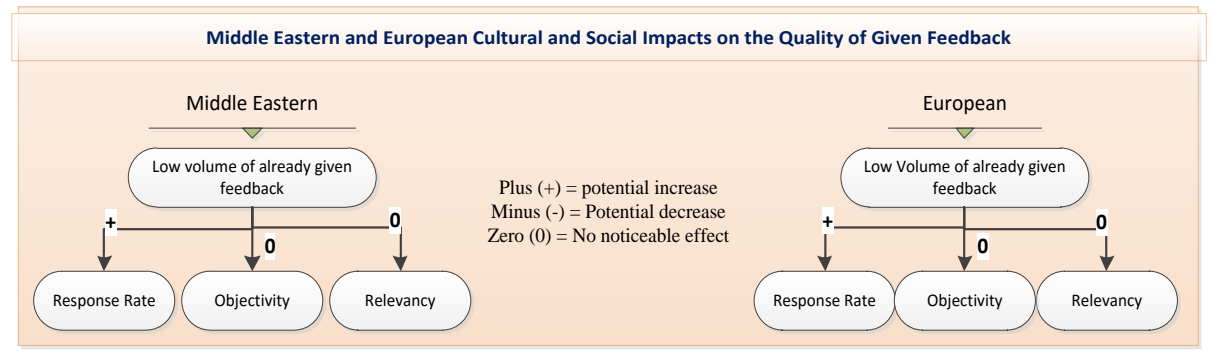

Fig. 5. The effect of feedback volume on feedback in Middle Eastern and European culture.

On the other hand, European users seemed to be far less motivated by the same aspect as few participants would have thought in a similar way. In fact, some of the European participants mentioned that some of the socially recognized feedback providers might have been selected and sponsored by the software/service provider for marketing reasons (e.g. being a celebrity). One of the participants commented "You know nowadays in advertisements they use popular names and faces to attract people. It can be the same case for feedback too". Although Middle Eastern users are also aware of this threat but they would still be more interested in the social aspect (social recognition) regardless of the potential threats it might cause. Generally, these potential threats could result in users having negative attitudes towards the provided software service or the product. Figure 6 gives a general view of how the different cultures' impact on this social factor could affect the quality of given feedback. In addition, the following dimensions of this theme were extracted from the participants' responses:

Feedback objectivity and relevancy. Social recognition as a motivating factor to feedback acquisition does not seem to play a role in affecting the objectivity and relevancy of feedback given by European users. In contrast to, the degree of relevancy and objectivity of feedback given by Middle Eastern users to the provided service might be slightly harmed. This is due to the fact that Middle Eastern users are more socially involved with their community and this can push them sometimes to act differently when they are being socially recognized as feedback providers. One of the participants commented "You know lots of people could be watching me. I will always try my best to be ideal in their eyes". This indeed could have negative impact on their given feedback such as imposing favouritism in their opinions regarding the provided software service which made them socially recognized at the first place. However, this social aspect could have a positive impact on the social behaviour within the online society of Middle Eastern users since socially recognized users would feel more socially constrained and their behaviour is always under the spot light of the community. This could result in an overall improved social behaviour and an online user community. 


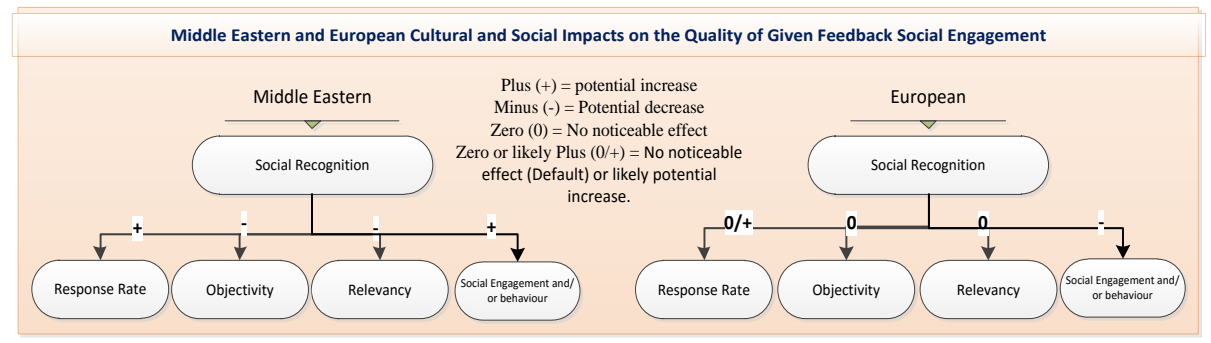

Fig. 6. The effect of social recognition on feedback in Middle Eastern and European culture.

Suitable and unsuitable uses of social recognition. As indicated by users' responses from both parties (Middle Eastern and European users) social recognition could be more beneficial and motivating if it is used in a small and closed community of users where all of the users somehow know each other (i.e. Facebook groups). This makes users more interested in being socially recognized to people who they know and more motivating for users to follow up a socially recognized person who they know and trust. One of the participants commented "It would be nice if we are all friends and know each other. I will trust and follow who I know and I will be happy to be recognized in front of people who care about me, right?"

On the other hand, users indicated that social recognition can lead to users following socially recognized users only and ignoring the feedback of unrecognized users even if their feedback is far better in quality. This of course could harm the provided software service and its reputation since vital information and knowledge about the provided service could be overlooked. In addition, some users mentioned that social recognition could lead to users' addiction to the used software especially young users who are eager to be socially recognized and more socially active. It is a trade-off between using this factor to benefit users or harm them and the provided service.

\subsection{Feedback acquisition as a social activity}

Similar to the above dimension, Middle Eastern users showed a much higher interest in conducting feedback acquisition as a social activity (i.e. social games) and emphasized that it would increase their willingness to give feedback. This was true especially for young users. Example of such an activity could be the users' ability to visualize how their direct and indirect social contacts are rating a certain service and how their feedback influenced the trend in their community. Compared to this, the majority of interviewed European showed a negative trend towards this factor. In fact, they believe feedback requests should be straightforward and simple by default and conducting it as a social activity could make the process of feedback acquisition more discouraging, complex and distracting from the main purpose which is evaluating a provided software service. One of the participants said in this regard "I think it should not look more than what it is supposed to do. It is to get your feedback and not a game to play with".

Feedback objectivity and relevancy. Conducting the feedback acquisition as a social activity could have a harmful effect on the objectivity and relevancy of the given feedback by Middle Eastern users. It is perhaps due to users' engagement with the activity more than the provided service as well as the burden of social constraints. One 
of the participants commented "I imagine I would be nicer about my opinion to people I know more than others". Although this might result in given feedback that does not reflect users' true opinion about the provided software service, it could also result in a more socially active users which could be a positive sign to the software and the users' community. Users' satisfaction with the social activity should not affect their view about the software service. This highlights the need to carefully design and apply this factor. Figure 7 gives a clearer view of how the different cultures' impact on this social factor could affect the quality of given feedback.

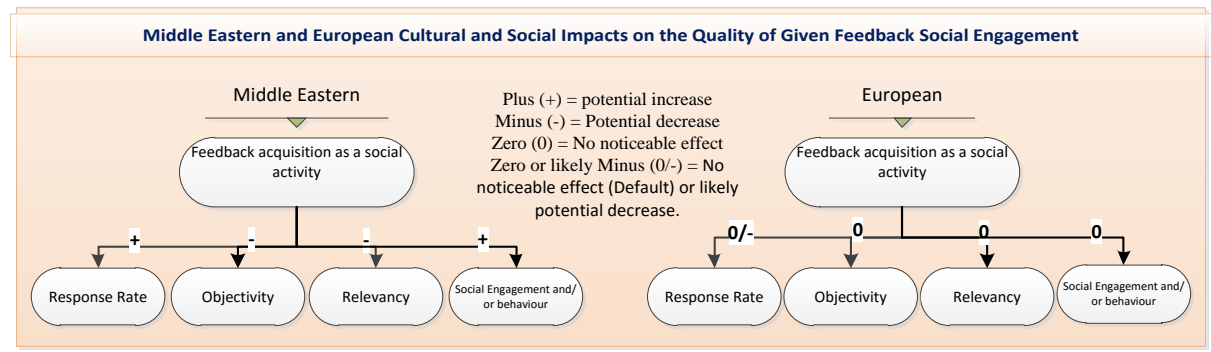

Fig. 7. Eeffect of feedback acquisition as a social activity in Middle Eastern and European culture.

In general, this paper advocated that culture variations has a noticeable impact on how Middle Eastern and European users' behaviour in response to feedback acquisition and how they are socially motivated to give feedback. The results suggest that having carfully tailopred persuasive method for the design of an adaptive and cultural-aware feedback acquisition could highly improve the quality of the collected feedback as well as users' satisfactions and response rate to feedback acquisition.

\section{Conclusion}

In this paper reported on an empirical study that investigated qualitatively the culture impact on users' behaviour and motivations to feedback acquisition. The result highlighted key differences between Middle Eastern and Western users with regard to their social motives to give feedback. Generally, European users found to be more sociallyindependent and showed less interest in being socially motivated to give feedback than Middle Eastern users. The paper also advocates the need to have a persuasive and culture-aware feedback acquisition which opens the gate for further research in this area.

\section{Acknowledgment}

We would like to acknowledge that this research was supported by an FP7 Marie Curie CIG grant (the SOCIAD Project). We would also like to thank the participants who took part in our study for their valuable input.

\section{References}

1. Yunker, J.:Beyond borders: Web globalization strategies. New Riders (2002). 
2. Reinecke, K. and Bernstein, A.: Culturally adaptive software: moving beyond internationalization. Usability and Internationalization. Global and Local User Interfaces. Springer, (2007)

3. Nielsen, J.: International user interfaces. Pfeil, U., Zaphiris, P. and Ang, C. S. Cultural differences in collaborative authoring of Wikipedia. Journal of Computer-Mediated Communication, 12 (1), (1996)

4. Honold, P.: Culture and context: An empirical study for the development of a framework for the elicitation of cultural influence in product usage. International Journal of HumanComputer Interaction, 12 (3-4), (2000) 327-345

5. Abdelnour-Nocera, J., Clemmensen, T. and Kurosu, M.: Reframing HCI Through Local and Indigenous Perspectives. International Journal of Human-Computer Interaction, (2013)

6. Raian Ali, Carlos Solís, Inah Omoronyia, Mazeiar Salehie, and Bashar Nuseibeh. Social adaptation - when software gives users a voice. In Joaquim Filipe and Leszek A. Maciaszek, editors, ENASE, pages 75-84. SciTePress, (2012)

7. Almaliki, M., Ncube, C. and Ali, R.: The design of adaptive acquisition of users feedback: An empirical study, RCIS, (2014)

8. Fogg, B. J.: Persuasive Technology: Using Computers to Change What We Think and Do. Morgan Kaufmann (2002)

9. Fogg, B. J., and Daisuke Iizawa.: Online persuasion in Facebook and Mixi: a cross-cultural comparison. In Persuasive technology, pp. 35-46. Springer Berlin Heidelberg (2008)

10. Khaled, Rilla.: Culturally-relevant persuasive technology (2008)

11. Marcus, A.: Global/intercultural user interface design. Human-Computer Interaction: Design Issues, Solutions, and Applications, 355-381 (2003)

12. Callahan, E.: Interface design and culture. Annual review of information science and technology, 39 (1)255-310 (2005)

13. Aykin, N., Quaet-Faslem, P. H. and Milewski, A. E.: Cultural ergonomics. Handbook of Human Factors and Ergonomics, Third Edition, 177-190 (2006)

14. Frandsen-Thorlacius, O., Hornbæk, K., Hertzum, M. and Clemmensen, T.: Non-universal usability?: a survey of how usability is understood by Chinese and Danish users, SIGCHI Conference on Human Factors in Computing Systems, ACM (2009)

15. Creswell, J. W., Plano Clark, V. L., Gutmann, M. L. and Hanson, W. E.: Advanced mixed methods research designs. Thousand Oaks, CA: Sage (2003)

16. Morse, J. M.: Approaches to qualitative-quantitative methodological triangulation. Nursing research, 40 (2), 120-123 (1991)

17. Tashakkori, A. and Teddlie, C.: Mixed methodology: Combining qualitative and quantitative approaches. Vol. 46. SAGE Publications (1998)

18. Creswell, J. W.: Qualitative Inquiry And Research Design: Choosing Among Five Approaches. 3rd. SAGE Publications, Inc (2013)

19. Socialclinic.: The State of Social Media in Saudi Arabia [online]. The Social Clinic. http://www.thesocialclinic.com/the-state-of-social-media-in-saudi-arabia-2012-2/ (2013)

20. Miles, M. B. and Huberman, A. M.: Qualitative data analysis: An expanded sourcebook. 2nd. Sage (1994)

21. Suler, J.: The online disinhibition effect. Cyberpsychology \& behavior, 7 (3), 321-326 (2004)

22. Almaliki, M.: Engineering an Adaptive and Socially-aware Feedback Acquisition. PhD thesis, Bournemouth University, 88-102 (2015) 